\title{
Comunicación

\section{Verdades a medias: la nominalización deverbal en los titulares periodísticos}

\author{
JUAN NADAL PALAZÓN1
}

La nominalización deverbal, entendida como el mecanismo de formación de sustantivos a partir de verbos, es un rasgo característico del lenguaje periodístico, especialmente en el discurso de los titulares. La elevada incidencia de estas formas responde a diversos factores -en su mayoría pragmáticos- que concurren en la enunciación, tales como la ideología subyacente y la economía lingüística. Destaca sobre todo la motivación ideológica, pues la elección de derivados nominales en lugar de oraciones completas revela, en muchos casos, la intención de omitir información relevante, como el agente o las circunstancias de la acción referida.

PALABRAS CLAVE: titulares, nominalización, reducción argumental, pragmática, ideología.
Deverbal nominalization, understood as the mechanism to make names out of verbs, is a characteristic feature of the journalistic language, especially within the headline's discourse. The high incidence of these forms responds to several factors (mainly pragmatic) that concur on the enunciation, such as the underlying ideology and the linguistic economy. The ideological motivation particularly stands out, since the election of nominal derivatives instead of complete sentences reveals, in many cases, the intention to omit relevant information, like the agent or the circumstances of the referred action.

KEY WORDS: headlines, nominalization, argument reduction, pragmatics, ideology.

1 Universidad Nacional Autónoma de México.

Correo electrónico: palazon@servidor.unam.mx 
¿Dijiste media verdad?

Dirán que mientes dos veces

si dices la otra mitad.

Antonio Machado

\section{INTRODUCCIÓN}

Toda forma de comunicación exige la presencia de un emisor. A su vez, toda forma de comunicación verbal presupone una realización individual de la lengua, lo cual implica, entre otras decisiones, la elección de estructuras gramaticales. Dado el inventario de posibilidades de que disponen los hablantes para expresarse, la elección de determinada estructura gramatical, y no otra, es una cuestión significativa desde el punto de vista de la pragmática integrada.

Las elecciones de estructuras gramaticales responden a motivaciones ideológicas (Fowler, 1986; Hodge y Kress, 1993 y Fairclough, 1995, entre otros): como bien ha dicho Van Dijk (2003: 68-69), "las ideologías subyacentes [...] afectan las estructuras formales del lenguaje". En concordancia con ello, debe entenderse que, en toda forma de codificación lingüística, el locutor orienta el sentido de su enunciado hacia determinadas interpretaciones (Ducrot [1972], 1982 y 1980, Anscombre y Ducrot, 1983). Ducrot afirma, desde esta perspectiva, que la lengua siempre da una aprehensión enunciativa del mundo, por cuanto la representación de la realidad pasa por el establecimiento previo de una serie de relaciones intersubjetivas dentro del discurso (Ducrot [1995], 1998: 124).

En cuanto actos de habla ( $c f$. Austin, 1962), las emisiones informativas poseen siempre un efecto perlocucionario. Si admitimos que la finalidad primordial del discurso periodístico es influir en el contenido y principios fundamentales de los conocimientos y representaciones sociales ( $c f$. Romero, 1996: 14), las configuraciones gramaticales características del discurso informativo deben responder a condicionamientos pragmáticos tendentes a imponer y reproducir posturas ideológicas ( $c f$. Van Dijk [1980], 1990 y 2003).

En este trabajo analizo una de las peculiaridades lingüísticas distintivas del discurso periodístico: la transformación nominal ( $c f$. , entre 
otros, Steel, 1971: 13; Casado Velarde, 1978: 103; Van Dijk [1980], 1990: 117 y Hurtado, 2003: 110), que más adelante definiré como el mecanismo de formación de sustantivos a partir de vocablos pertenecientes a otras categorías gramaticales. Me baso en un corpus de 2397 titulares, publicados en su mayoría durante febrero de 2005 en los diarios mexicanos Reforma, El Universal, El Sol de México, La Jornada y La Prensa.

Como se sabe, "los titulares son la parte más importante del texto" periodístico (Van Dijk [1988], 1997: 134), pues, además de sintetizar el texto que encabezan, orientan su interpretación ( $c f$. Eco, 1977:165-187, Van Dijk [1980], 1990, [1988] 1997, 2006, Thogmartin, 1991:249-266). En muchos casos, sin embargo, su importancia es aún mayor: constituyen las únicas secuencias leídas por gran parte de los consumidores de diarios. No en vano el titular es, para Steel (1971: 15), "la forma periodística por antonomasia".

Me propongo demostrar en las líneas siguientes que el uso de nominalizaciones deverbales en los titulares periodísticos encuentra su motivación en razones de índole pragmática, relacionadas sobre todo con la ideología del locutor y con el principio general de economía lingüística. Mi exposición responde al orden siguiente: en primer lugar, defino el concepto de nominalización deverbal y proporciono una revisión muy general de algunos de los estudios que al respecto se han publicado; en segundo, refiero las características de la nominalización deverbal en los titulares periodísticos, así como los condicionamientos pragmáticos que mis materiales revelan. Cierran unas conclusiones.

\section{LAS NOMINALIZACIONES DEVERBALES}

El término nominalización ${ }^{2}$ alude al proceso de formación de sustantivos o nombres a partir de una base perteneciente a otra categoría gramatical. Es, por tanto, un mecanismo de derivación. ${ }^{3}$ Esto ocurre,

2 “Formación sustantiva” para Alvar y Pottier (1983: 383).

3 Derivación: en términos generales, proceso morfológico de formación de léxico (Matthews 1980: 50) en el que hay adición, supresión o intercambio de sufijos ligados a una forma libre. Conlleva una alteración en el signifi- 
en español, gracias a la adición de sufijos a bases léxicas verbales o adjetivales. Así, por ejemplo, se obtiene el sustantivo recaudación al añadir el sufijo - (a)ción a la base del verbo recaudar, o el sustantivo simplismo, mediante la adición de -ismo a la base del adjetivo simple. Cuando la base a la que se adjunta el sufijo nominalizador es de naturaleza verbal, la nominalización se especifica como deverbal. En cambio, se califica como deadjetival cuando corresponde a un adjetivo la unidad que sirve de base para la derivación nominal. 4

En esta ocasión profundizaré únicamente en ciertas nominalizaciones deverbales: trataré aquellas que suelen definirse como "acción y efecto" y que Comrie (1976: 178) llamó "nombres de acción" (action nominals). Las partículas -(a)ción, -sión, -ión, -miento, -aje y -ura son algunos de los morfemas nominalizadores de acción que tiene la lengua española ( $c f$. Miranda, 1994: 137 y ss.). En el siguiente cuadro reúno varios ejemplos:

\begin{tabular}{llll} 
Base verbal & \multicolumn{1}{c}{$\begin{array}{c}\text { Sufijo } \\
\text { nominalizador }\end{array}$} & $\begin{array}{c}\text { Derivado } \\
\text { nominal }\end{array}$ & \multicolumn{1}{c}{ Significado } \\
\hline $\begin{array}{l}\text { Grabar } \\
\text { Aprehender }\end{array}$ & - sión & $\begin{array}{l}\text { Grabación } \\
\text { Aprehensión }\end{array}$ & $\begin{array}{l}\text { 'Acción y efecto de grabar' } \\
\text { 'Acción y efecto } \\
\text { de aprehender' }\end{array}$ \\
Unir & - ión & Unión & 'Acción y efecto de unir' \\
Acercar & - miento & Acercamiento & 'Acción y efecto de acercar' \\
Camuflar & - aje & Camuflaje & 'Acción y efecto de camuflar' \\
Clausurar & - ura & Clausura & 'Acción y efecto de clausurar' \\
Maltratar & $-o$ & Maltrato & 'Acción y efecto de maltratar'
\end{tabular}

cado de la base y, en ocasiones, también un cambio de categoría gramatical (Varela 1992: 70). Los morfemas derivativos tienden a ser numerosos, pero suelen ocurrir en pocas combinaciones (Nida, 1949: 99), a diferencia de los morfemas flexivos.

4 La voz nominalización también se ha hecho extensiva a la formación de nombres a partir de bases catalogadas, a su vez, como nominales: algunos autores llaman nominalizaciones denominales a derivaciones del tipo estoque > estocada. 
En los inicios de la gramática generativa, las nominalizaciones eran vistas como resultados de transformaciones (Chomsky, 1957); posteriormente, Chomsky (1970:184-221) propuso, en contra de esta idea, la "hipótesis lexicalista de la derivación de nominalizaciones de acción", según la cual estas constituyen entradas separadas en el diccionario (basa su argumentación en las semejanzas sintácticas que en inglés existen entre estos derivados y los nombres no derivados). Años después, desde una perspectiva tipológica y luego de analizar datos en ruso, polaco, búlgaro, macedonio, árabe clásico, turco y uzbeko, Comrie (1976:177201) determinó que lo importante es el grado de correspondencia que hay entre la estructura interna de una nominalización de acción y la de una frase nominal no derivada, atendiendo al modo como se presentan los argumentos, sujetos y objetos 5 .

Para Halliday (1994: 352), la nominalización es el "más poderoso recurso para crear metáforas gramaticales"6. En toda metáfora gramatical un componente semántico se construye en la gramática de manera no prototípica. Halliday propone dicho término para referirse al "mismo significado" que se evoca por medio de otra clase de palabra, donde se produce una conjunción de dos niveles de significado como resultado de una nueva elección gramatical. Así, gracias a la nominalización de acciones, los procesos, normalmente expresados con verbos, se reformulan metafóricamente como sustantivos: según Koptjevskaja-Tamm (1993: 6), las formaciones sustantivas ocupan una posición intermedia entre los verbos y los sustantivos típicos, y comparten rasgos semánticos y discursivos con ambos.

En el mismo sentido, de acuerdo con Fowler (1986), la nominalización es un proceso organizativo del mensaje que reduce una oración a un nombre; de este modo, se aminora la transitividad y se pierde el conocimiento de los participantes personales. Por ejemplo, la palabra admisión esconde referencias del tipo "yo admito a usted", en enuncia-

5 Koptjevskaja-Tamm (1993) ha adoptado la postura de Comrie y ha intentado establecer una tipología sintáctica de las nominalizaciones deverbales, con base en el análisis de un corpus con datos de setenta lenguas.

6 "Nominalizing is the single most powerful resource for creating grammatical metaphor" (Halliday, 1994: 352). 
dos como "la admisión de los aspirantes está sujeta a la disponibilidad de plazas", y muestra el proceso de admitir algo como estático, casi como si fuera un objeto.

\section{LAS NOMINALIZACIONES DEVERBALES}

EN LOS TITULARES PERIODÍSTICOS

Y SUS CONDICIONAMIENTOS PRAGMÁTICOS

Por todos conocida es la tendencia nominalizadora del discurso periodístico: Steel (1971: 13), por ejemplo, apunta con razón que, en este, “el grupo nominal adquiere una relevancia y una extensión mayor que en otros estilos de lengua" ( $c f$. Van Dijk [1980], 1990: 117, Hurtado, 2003: 110). Casado Velarde (1978: 103) destaca, por su parte, el reiterado uso de formaciones sustantivas en la confección de encabezados: "Con gran frecuencia en los titulares se presenta de forma nominalizada el hecho que se expresa con un verbo en el cuerpo de la noticia". Contiene derivados nominales $66.3 \%$ (1589/2397) de los titulares que integran mi corpus.

La elevada incidencia de nominalizaciones de acción puede explicarse, en principio, por la indudable necesidad de ahorrar espacio o tiempo en los medios: cuando se usa el código metafórico, no sólo se está interesado en incorporar más información, sino en hacerlo de manera más económica. Esto es evidente en los encabezados, sobre todo si recordamos que la codificación lingüística de estos es el resultado de un complejo proceso en el que incide, de manera definitiva, la tensión establecida por lo que Grice (1975) llamó la "máxima de cantidad", es decir, el principio lingüístico que apunta a no expresar ni más ni menos información que la estrictamente requerida por los objetivos del intercambio en curso.

(1) a. Silencia Cuba el arribo del nuevo embajador soviético [JOR 12/12/91: 51]

b. Impugna Navarrete actuación del IEEM sobre presuntas facturas falsas del PAN [LAP 23/02/05: 8] 7

7 En todos los encabezados reproducidos en este trabajo he respetado fielmente la ortografía literal y puntual de los originales. He conservado también los usos de mayúsculas y minúsculas. Asimismo, he transcrito los 
En muchos casos de nominalización de verbos intransitivos -y en algunos de transitivos-, todos los participantes aparecen expresados mediante modificaciones al núcleo sustantivo. Me refiero a casos como los de la serie (1), donde complementos adnominales 8 refieren los agentes de las acciones expresadas por medio de nominalizaciones deverbales: en el ejemplo (1a), el nuevo embajador soviético es quien arriba, y en (1b), el Instituto Electoral del Estado de México es quien actúa. Se trata, desde luego, de casos de motivación puramente económica, como evidencia su cotejo con las secuencias de la serie (1'), integrada por construcciones hipotéticas:

(1') a. Arriba el nuevo embajador soviético a Cuba, y el gobierno de la Isla lo silencia

b. El IEEM actúa con respecto a presuntas facturas falsas del PAN, y Navarrete impugna

Las construcciones bajo (1') son, obviamente, más extensas que los enunciados de la serie (1). Los casos de (1') revelan que, en la enunciación de titulares de (1), se asume la existencia ciertas oraciones, cuyos contenidos se expresan, con un valor semántico de contenido presupuesto, en grupos nominales que ejercen el oficio sintáctico de complemento directo. Secuencias como las de la serie (1') difícilmente se publicarían como titulares periodísticos, no sólo por su escasa economía lingüística, sino también porque las oraciones en posición focal

encabezados en letras de espesor regular, bien entendido que los titulares siempre se imprimen en negrillas, cuyo uso se reserva aquí para destacar algunas palabras a las que se alude en el análisis. Entre corchetes añado la referencia del texto: coloco primero el nombre del periódico, abreviado y en versalitas; después de un espacio, la fecha, y, por último, tras dos puntos, la página. Al final de este trabajo se encuentra la correspondiente lista de abreviaturas.

8 Un complemento adnominal es un sintagma nominal o pronominal que determina, por medio de una preposición, a un núcleo sustantivo, adjetivo o adverbial. En los casos que ahora nos interesan, ese núcleo es la nominalización deverbal. 
-es decir, al principio- refieren, en estos casos, información consabida en el momento del acto de habla: se trata, claro está, de contenidos que aluden anafóricamente a otras ediciones del diario.

Las nominalizaciones de acción, sin embargo, suelen comportar la ausencia de información argumental. 9 Esto puede deberse a dos razones: a que el periodista ignora la información o bien a que decide omitirla. Por ejemplo, en (2a) parece claro que el locutor ignora quién es el agente de la acción nominalizada, y que considera irrelevante la identidad de las víctimas, acaso por conocida o genérica. En (2b), en cambio, parece que la generalización inherente al contenido noticioso seleccionado ocasiona la omisión de los numerosos agentes del maltrato infantil.

(2) a. Preocupa en León ola de asesinatos [SOL 24/02/05: 8/B]

b. Niñas, víctimas del 95 por ciento de maltrato infantil [SOL 21/02/05: 10/A]

En otros encabezados, la decisión de no mencionar información argumental responde a motivaciones de índole ideológica. En conformidad con el modelo del cuadrado ideológico, de Van Dijk (2003), el acto de callar o expresar información conocida casi siempre encuentra su motivación en la ineludible existencia de una ideología subyacente; en el discurso de los medios de comunicación, ello supone, según este autor (2006), la imposición autoritaria de una visión del mundo.

Al igual que Van Dijk, Hodge y Kress (1993: 19 y ss.) consideran que es posible advertir algunos aspectos de la ideología del hablante si se atiende a la mención u omisión que este hace de los participantes que normalmente están unidos por un proceso verbal: con las omisiones, concebidas por estos últimos como mecanismos de un modelo de producción textual llamado "no transaccional" (non transactive model), se producen enunciados comprensivos de una sola entidad del proceso

9 Procedente de la lógica de predicados, la denominación argumento se utiliza en gramática para referirse a cada uno de los sintagmas nominales que un predicado relaciona y que son requeridos por este para efectuar una predicación completa. 
aludido, la cual no siempre coincide con el agente o el experimentador. Se trata, según Fowler (1986), de procesos organizativos del mensaje que responden a pérdidas de interés informativo.

(3) a. Cobija operativo extorsión [REF 23/02/05: 8B]

b. Por primera vez, pago íntegro a 18,000 productores de frijol [LAP 21/02/05: 44]

c. En mayo, clausura definitiva de dos tiraderos en Nezahualcóyotl [SOL 25/02/05: 5/A,3.']

d. Finaliza la identificación de víctimas del 11 de septiembre [JOR 24/02/05: 33]

En los casos (3a) a (3d), encontramos las voces extorsión, pago, clausura e identificación, respectivamente. En todos estos encabezados, la nominalización oculta información argumental muy importante desde el punto de vista informativo: ¿quién extorsiona a quién?, ¿quién paga cierta suma íntegra a 18 mil productores de frijol?, ¿quién clausura definitivamente dos tiraderos en Nezahualcóyotl?, ¿quién identifica a las víctimas del 11 de septiembre?

No parece que este tipo de déficit informativo responda a la intención de causar un extrañamiento tal en el alocutario que lo invite a consumir íntegramente el texto que el titular encabeza: a falta de información argumental, temporal, modal, aspectual y circunstancial, el proceso nominalizado se presenta la mayoría de las veces como si fuera espontáneo, a pesar de tratarse de derivados de formas léxicas cuya estructura semántica exige o presupone la existencia de entidades animadas que instigan, controlan y dirigen la acción representada.

El efecto de ambigüedad en la nominalización de acciones se debe a la habitual pérdida de argumentos y circunstancias, a la vez que de tiempo, aspecto, modo y modalidad verbales. El uso de esta metáfora gramatical apunta a un texto que, además de ambiguo, es estático: al convertir un proceso verbal en sustantivo, es decir, al mostrar una acción como si fuera un objeto, la relación entre procesos y participantes se muestra inmóvil. Sin duda, estas propiedades son aprovechadas -consciente o inconscientemente- por los redactores de titulares para restar importancia a los agentes, que por lo general desempeñan una 
función sujetiva y, por tanto, cardinal en la estructura profunda del enunciado.

La omisión de algunos segmentos en el uso de estas entidades abstractas implica que el lector deba completar la información faltante en la compleja fase de interpretación del titular, proceso que, como se sabe, no suele tomar en cuenta el texto que el encabezado rotula ( $c f$. Emig, 1927:53-59, Eco, 1977:165-187, Casado Velarde, 1984:235-242, Hurtado, 2003). Hodge y Kress (1993) consideran que la estructura profunda no siempre puede ser recobrada en su totalidad, por lo que los receptores frecuentemente interpretan los enunciados de maneras muy diversas: "El escritor presumiblemente conoce con exactitud el significado pleno, pero quizá el lector no",10 advierte Halliday (1994: 353).

Analicemos más detalladamente el caso (3b). En este encabezado encontramos la forma pago, que constituye un derivado nominal del verbo pagar. ${ }^{11}$ Sintácticamente, esta palabra constituye el núcleo del sujeto de una oración simple de predicado adverbial con verbo elíptico. Desde un punto de vista semántico, la "acción de pagar" recibe tres especificaciones en el contexto lingüístico: una temporal-aspectual (A), que apunta a que nunca antes había ocurrido un suceso análogo; una modal (B), que detalla la naturaleza integral del pago, y una alusiva al supuesto paciente de la acción referida $(\mathrm{C})$, es decir, los 18 mil productores de frijol.

$\underline{\text { Por primera vez, pago íntegro a } 18,000 \text { productores de frijol }}$

A

B

$\mathrm{C}$
A: Especificación temporal-aspectual
B: Especificación modal
C: Especificación de paciente

Como hemos dicho, el titular no especifica el agente del proceso: no sabemos quién pagó, por primera vez, una cierta cantidad íntegra a

10 "The writer presumably knows exactly what it means; but the reader may not".

11 Productores es también una nominalización, pero de momento no nos interesa. 
18 mil productores de frijol. Una lectura a la nota informativa permite saber que se trata de la Secretaría de Agricultura, la cual, además, resulta ser el origen de la información procesada. Desde una perspectiva pragmática, esto revela la clara intención de restar importancia al agente de una acción cuya referencia presenta, en principio, connotaciones positivas. Bien sugiere Van Dijk (2003) que el omitir o disimular información relacionada con logros ajenos supone rivalidades o posturas discordantes con relación a la entidad responsable de estos.

Esta interpretación es comprobable por el hecho de que se enfatiza la especificación temporal-aspectual del derivado nominal, esto es, el contenido expuesto que denota la naturaleza inaugural de la acción. Situada en posición focal, es decir, al principio del encabezado, esta información supone que las autoridades agrarias no han cumplido, históricamente, con algo que el contexto presenta como una obligación gubernamental. Apoya esta lectura la especificación modal, de la cual se deriva la conclusión de que los pagos anteriores han sido incompletos. Todo esto presenta a los 18 mil campesinos, más que como beneficiarios de la acción nominalizada, como víctimas del abuso de las autoridades correspondientes.

\section{CONCLUSIONES}

Primera: la nominalización deverbal es un fenómeno muy frecuente en los titulares periodísticos. Su uso puede y debe ser estudiado con métodos lingüísticos de probada validez, como muchas otras singularidades del discurso informativo verbalmente codificado.

Segunda: en los titulares periodísticos el uso de nominalizaciones deverbales responde a diversos factores -en su mayoría pragmáticos-, que concurren en la enunciación, tales como la ideología subyacente, la adaptabilidad del lenguaje y el principio de economía lingüística.

Tercera: la complejidad de los titulares periodísticos es, frecuentemente, el resultado de una alta densidad léxica y de una fuerte nominalización, en la que los procesos verbales están metafóricamente codificados como estructuras nominales. Tal situación se explica por el hecho de que la nominalización permite reducir oraciones completas a sustantivos que se incorporan de manera normal a cualquier estructura sintáctica. 
Cuarta: la nominalización de acciones suele producir un efecto de ambigüedad en el enunciado. Esto se debe a que normalmente hay pérdidas de información argumental, circunstancial, temporal, modal y aspectual; la omisión del agente suele dar por resultado que el proceso referido parezca espontáneo. Además, el convertir un proceso verbal en sustantivo ocasiona que la relación entre procesos y participantes se muestre inmóvil.

Quinta: la elección de derivados nominales en lugar de oraciones completas revela, en muchos casos, la existencia de una estrategia persuasiva que comporta la omisión de datos relevantes, como el agente o las circunstancias de la acción referida. Por cuanto el uso de estas formas suele ser una consecuencia de la actitud que el periodista adopta ante los contenidos informativos durante el proceso de construcción de la noticia, el análisis de este tipo de "verdades a medias" devela posturas ideológicas y, por ende, líneas editoriales.

\section{Bibliografia}

AlarCOS LloraCH, Emilio (1977) "Lenguaje de los titulares", en Lenguaje en periodismo escrito, F. Lázaro Carreter (ed.). Madrid: Fundación Juan March (Serie Universitaria, 37).

AlVAR, Manuel y Bernard Pottier (1983) Morfología histórica del español. Madrid: Gredos (Biblioteca Románica Hispánica, III: Manuales, 57).

AN SCOMBRE, Jean-Claude y Oswald Ducrot (1983) L'argumentation dans la langue. Bruxelles: Pierre Mardaga.

AUstin, John Langshaw. 1962 How to do things with words. Cambridge: Harvard University Press.

CASADO VELARDE, Manuel (1978) "La transformación nominal, un rasgo de estilo de la lengua periodística", Cuadernos de Investigación Filológica, 4 (1).

- (1984) "Semiótica de los titulares: pautas para el análisis de los titulares periodísticos", en Teoría semiótica. Actas del Congreso Internacional sobre Semiótica e Hispanismo, vol. 1, M. Á. Garrido Gallardo (ed.). Madrid: CSIC.

CHOMSKY Noam (1957) Syntactic structures. The Hague: Mouton.

- (1970) "Remarks on nominalization", en Readings in english trans- 
formational grammar, R. Jacobs y P. Rosenbaum (eds.). Waltham: Ginn.

COMRIE, Bernard (1976) "The syntax of action nominals: a cross-language study", Lingua, 40.

DuCROT, Oswald (1972) (1982) Decir y no decir. trad. Walter Minetto y Amparo Hurtado. Barcelona: Anagrama (Biblioteca de Lingüística, 2).

- (1980) Les échelles argumentatives. Paris: Minuit.

- (1995) (1998) "Componentes de la descripción lingüística", en Nuevo diccionario enciclopédico de las ciencias del lenguaje, $\mathrm{O}$. Ducrot y J. M. Shaeffer (eds.). trad. María Camino Girón, Teresa María Rodríguez y Marta Tordesillas. Madrid: Arrecife.

ECO, Umberto (1977) "Guida all'ipretazione del linguagio giornalistico", en La lingua italiana oggi: un problema scolastico e sociale, L. Renzi y M. A. Cortelazzo (eds.). Bologna: Società Editrice il Mulino (problemi e prospective. Serie di linguistica e critica litteraria).

EMIG, Elmer (1927) “The connotation of newspaper headlines”, Journalism Quarterly, 4 (4).

FAIRCLOUGH, Norman (1995) Media discourse. London: Edward Arnold.

FOWLER, Roger. (1986) Linguistic criticism. Oxford: Oxford University Press.

GRICE, Herbert Paul (1975) "Logic and conversation", en Syntax and Semantics 3: Speech Acts, P. Cole y J. L. Morgan (eds.). New York: Academic Press.

Halliday, Michael Alexander Kirkwood (1994) An introduction to functional grammar. 2. ${ }^{\mathrm{a}}$ ed. London: Edward Arnold.

HODGE, Robert y Gunther Kress (1993) Language as ideology. 2. ${ }^{\mathrm{a}} \mathrm{ed}$. New York: Routledge.

Hurtado GonzÁlez, Silvia (2003) El uso del lenguaje en la prensa escrita. Valladolid: Universidad de Valladolid (Lingüística y Filología, 56).

KOPTJEVSKAJA-TAMM, Maria (1993) Nominalizations. LondonNew York: Routledge (Theoretical Linguistics).

MACHADO, Antonio (1899-1930) (1964) Obras. Poesía y prosa. Re- 
cop. Aurora de Albornoz y Guillermo de la Torre. Buenos Aires: Losada.

MALCHUKOV, Andrej L. (2004) Nominalization/verbalization: constraining a typology of transcategorial operations. München: Lincom (Studies in Language Typology, 8).

MattheWs, Peter H. (1980) Morfología. Introducción a la teoría de la estructura de la palabra. Trad. Rafael Monroy Casas. Madrid: Paraninfo.

NADAL PALAZÓN, Juan (2007) "El estilo directo no marcado en los titulares de la prensa mexicana", en X Simposio Internacional de Comunicación Social, Actas-I, L. Ruiz, A. Muñoz y C. Álvarez (eds.). Santiago de Cuba: Centro de Lingüística Aplicada.

NIDA, Eugene A. (1949) Morphology. The descriptive analysis of words. 2. ${ }^{\mathrm{a}}$ ed. Ann Arbor: The University of Michigan Press.

Romero Álvarez, María de Lourdes (1996) "El relato periodístico como acto de habla", Revista Mexicana de Ciencias Políticas y Sociales, 169.

STEEL, Brian (1971) "Los estilos funcionales y la enseñanza del idioma”, Español Actual.

THOGMARTIN, Clyde (1991) "The pragmatics of french newspaper headlines", en Levels of linguistic adaptation, J. Verschueren (ed.). Amsterdam-Philadelphia: John Benjamins (Pragmatics \& Beyond, New Series, 6: 2).

VAN DIJK, Teun Adrianus [1980] (1990) La noticia como discurso. Trad. Guillermo Gal. Barcelona: Paidós (Paidós Comunicación, 41).

- (1988) (1997) “Cómo se lleva una minoría a los titulares? Minorías étnicas en la prensa", en Racismo y análisis crítico de los medios. Trad. Montserrat Basté Kraan. Barcelona: Paidós (Paidós Comunicación, 82).

- (2003) Ideología y discurso. Barcelona: Ariel (Ariel Lingüística).

- (2006) "Discurso y manipulación: discusión teórica y algunas aplicaciones". Trad. Marianne Peronard. Signos, 60.

Varela Ortega, Soledad (1992) Fundamentos de morfología. Madrid: Síntesis (Lingüística, 5). 


\section{Abreviaturas}

$$
\begin{aligned}
& \mathrm{JOR}=\text { La Jornada } \\
& \mathrm{LAP}=\text { La Prensa } \\
& \mathrm{REF}=\text { Reforma } \\
& \mathrm{SOL}=\text { El Sol de México } \\
& \mathrm{UNI}=\text { El Universal }
\end{aligned}
$$

